\title{
Authors response to comment of Prof. Dr. Amid on original article "Overlap-coefficient for the relationship between mesh size and defect size in ventral hernia surgery"
}

\author{
J. Lambrecht
}

Published online: 24 May 2011

(C) Springer-Verlag 2011

\section{Dear Editors,}

I am honored that my proposals are being reviewed by Professor Amid and I certainly agree with Professor Amid that the proposed coefficient is not applicable to all methods of repair. I profoundly excuse my single-mindedness on laparoscopic repair, and especially apologize that my manuscript did not state that the proposal is with this method of repair in mind.

My only disagreements to the comments of Professor Amid is the restriction on defect size to the laparoscopic technique, and the popularity-ranking. The laparoscopic approach is only partly restricted to loss-of-domain and extensive adhesion, and may be contraindicated in radiated patients where one should consider a stringent extraperitoneal approach to best avoid the risk of fistulae. However, in cases with extensive adhesion, where laparoscopic dissection is deemed to be too risky, many surgeons in my part of the world (myself included) still prefer an intraperitoneal onlay mesh (IPOM) placed by laparoscopy, even after the rare necessity of open reduction and adhesiolysis. Hernia site or size is really not an issue-laparoscopic repair can be attractive also in very large hernias-and more so in "extreme" locations.

I am aware of peer-reviewed publications with recommended size restrictions for the laparoscopic repair. There are more publications without such restrictions. My own experience includes non-publicized data from two studies: one retrospective in 92 repairs, but with clinical examination (and ultrasonography [US] when in doubt) after

J. Lambrecht $(\bowtie)$

Sykehuset Innlandet Medical Trust, Kyrre Grepsgate 11,

2819 Gjoevik, Norway

e-mail: jan.lambrecht@sykehuset-innlandet.no;

jan@lambrecht.no
3 years of follow-up specifically for the control of hernia repair, and one randomized multicenter study with 156 repairs, comprising 34 immune compromised kidney or liver transplanted patients, where inclusion was stopped 1.5 years ago after 3 years of inclusion. In neither of these datasets are there, so far, any correlation between size and recurrence, even in hernia sizes over $10 \mathrm{~cm}$ (up to $25 \mathrm{~cm}$ ), but there is a correlation between size and pain. Unfortunately, I have, so far, not found a correspondence between the proposed overlap-coefficient and recurrence, which might insinuate that our targeted 5-cm overlap is more than sufficient or the dataset too small.

Even though all publicized studies on laparoscopic repair have data on defect size and mesh size, the significance of overlap has not been extensively studied. The proposed coefficient could be applied to these datasets, with different "set points" to evaluate overlap in respect of recurrence or mesh protrusion, in order to find a more communicative figure for the required overlap. Of course, there are many other factors implicated. such as mesh shrinkage, mesh in-growth capability and flexibility, fixation methods, removal of intrafascial fat, and accuracy in the application. It would still be a joy for me to hear reports from datasets with estimates of complications also founded on, e.g., the proposed coefficient—or a better and maybe universally applicable manner of analysis-as Professor Amid rightfully desires. My best hope is that the many more-than-I academically minded peers might be inspired to use a variant of the proposed coefficient or find a better way for this type of analysis.

I thank you for considering my proposal. 